\title{
Radiography, computed tomography and magnetic resonance imaging at 0.5 Tesla of mechanically induced osteoarthritis in rabbit knees
}

S.R. Torelli1, S.C. Rahal2, R.S. Volpi ${ }^{4}$, S. Yamashita ${ }^{5}$, M.J. Mamprim ${ }^{3}$ and A.J. Crocci ${ }^{6}$

\section{Correspondence}

S.C. Rahal

Departamento de Cirurgia e

Anestesiologia Veterinária

Faculdade de Medicina Veterinária

e Zootecnia, UNESP

Rubião Júnior, $s / n$

18618-000 Botucatu, SP

Brasil

E-mail: sheilacr@fmvz.unesp.br

Research supported by FAPESP.

Received February 27, 2003

Accepted January 21, 2004
1Programa de Pós-graduação em Medicina Veterinária, and Departamentos de ${ }^{2}$ Cirurgia e Anestesiologia Veterinária, and ${ }^{3}$ Reprodução Animal e Radiologia, Faculdade de Medicina Veterinária e Zootecnia, Universidade Estadual Paulista, Botucatu, SP, Brasil

Departamentos de ${ }^{4}$ Cirurgia e 0 rtopedia and ${ }^{5} \mathrm{D}$ oenças Tropicais e Diagnóstico por Imagens, Faculdade de Medicina de Botucatu, Universidade Estadual Paulista, Botucatu, SP, Brasil

${ }^{6}$ Departamento de Bioestatística, Instituto de Biociências, Universidade Estadual Paulista, Botucatu, SP, Brasil

\section{Abstract}

In the present experimental study we assessed induced osteoarthritis data in rabbits, compared three diagnostic methods, i.e., radiography (XR), computed tomography (CT) and magnetic resonance imaging (MRI), and correlated the imaging findings with those obtained by macroscopic evaluation. Ten young female rabbits of the Norfolk breed were used. Seven rabbits had the right knee immobilized in extension for a period of 12 weeks (immobilized group), and three others did not have a limb immobilized and were maintained under the same conditions (control group). Alterations observed by XR, CT and MRI after the period of immobilization were osteophytes, osteochondral lesions, increase and decrease of joint space, all of them present both in the immobilized and non-immobilized contralateral limbs. However, a significantly higher score was obtained for the immobilized limbs (XT: $\mathrm{P}=0.016, \mathrm{CT}: \mathrm{P}=0.031$, MRI: $\mathrm{P}=0.0156$ ). All imaging methods were able to detect osteoarthritis changes after the 12 weeks of immobilization. Macroscopic evaluation identified increased thickening of joint capsule, proliferative and connective tissue in the femoropatellar joint, and irregularities of articular cartilage, especially in immobilized knees. The differences among XR, CT and MRI were not statistically significant for the immobilized knees. However, MRI using a 0.5 Tesla scanner was statistically different from CT and XR for the non-immobilized contralateral knees. We conclude that the three methods detected osteoarthritis lesions in rabbit knees, but MRI was less sensitive than XR and CT in detecting lesions compatible with initial osteoarthritis. Since none of the techniques revealed all the lesions, it is important to use all methods to establish an accurate diagnosis.

\section{Key words}

- O steoarthritis

- Rabbit knee osteoarthritis

- Imaging diagnosis

- Knee immobilization

- Radiography

- Computed tomography

- Magnetic resonance imaging 


\section{Introduction}

Joint movement restriction is one of a variety of procedures that can be used to experimentally induce changes in the knee articular cartilage (1). Immobilization results in degenerative articular alterations in both splinted and contralateral knee joints (2), with abnormal proliferating cartilage observed peripherally toward the edges of the joint cartilage (3). Osteophyte formation is observed after 14 days of immobilization (3).

Radiography (XR) is the method most frequently used to evaluate alterations in the musculoskeletal system (4). It can be used to detect hard and soft tissue changes in early stages of osteoarthritis, but multiple radiographic projections are necessary (5). Computed tomography (CT) is an accurate examination technique for osteoarthritis lesions, which most frequently involve the knee (6). It provides excellent contrast resolution and permits differentiation of soft tissue structures that cannot be seen precisely by conventional radiography (4). Magnetic resonance imaging (MRI) is a sensitive imaging modality for the internal anatomy of the knee joint without the need for any type of contrast agent or joint manipulation (7).

The aim of the present study was to compare the efficacy of XR, CT and MRI for the diagnosis of osteoarthritis induced by continuous immobilization in rabbits, and to correlate the imaging findings with macroscopic evaluation used as a "gold" standard.

\section{Material and Methods}

The Ethics Committee of the Faculty of Veterinary Medicine and Animal Science, UNESP, Botucatu, approved the study.

Ten female Norfolk rabbits, initial age from 2.5 to 3 months and initial weight 2.5 $\mathrm{kg}$, housed in individual cages of standard size were used. The animals received commercial food and water ad libitum. Seven rabbits had the right knee immobilized in extension for a period of 12 weeks to induce degenerative joint disease (immobilized group) and three others were not immobilized (control group). The method used to immobilize the knee was an individually shaped PVC splint applied to the caudal aspect of the right leg from the proximal end of the thigh to the distal end of the limb (2,3,8-10). Rabbits were premedicated with acepromazine $(1.0 \mathrm{mg} / \mathrm{kg}, \mathrm{im})$ and anesthetized with sodium pentobarbital $(15 \mathrm{mg} / \mathrm{kg}$, $i v$ ), and both knees were evaluated by $\mathrm{XR}$ (TUR D800, Germany), CT (GE-SYTEC2001, Japan) and MRI (GE-MR-MAX, 0.5 Tesla, Japan), before immobilization (TI) and 12 weeks after immobilization (TII). Radiographic examinations were performed at our Veterinary Hospital, and CT and MRI in the Imaging Diagnostic Service of the Faculty of Medicine, UNESP, Botucatu. After 12 weeks, the animals were sacrificed for macroscopic examination.

Radiographs were obtained in lateral, craniocaudal and skyline projections. XR features observed were osteophyte formation, femoral condyle subchondral lesions, tibial subchondral lesions, femoral condyle irregularity, femoral osteochondral reaction, and increase or decrease of articular space.

For the CT study, animals were positioned in dorsal decubitus with their legs extended. Both knees were studied simultaneously, without any contrast. A scout anteroposterior view was obtained, and the boundaries of the CT scan were determined by placing an initial cursor proximal to the femoropatellar joint and a final cursor over the tibial tubercle. CT sections were obtained using a transverse 5$\mathrm{mm}$ thick plane with a 5-mm intersection gap. The machine parameters were $120 \mathrm{kV}$ and 80 $\mathrm{mA}$, with $3.6 \mathrm{~s}$ of scan time for bone and soft tissue windows. The CT changes evaluated included radiological signs of femoral condyle osteochondral lesion, tibial osteochondral lesion, fibular osteochondral lesion, femoropatellar joint osteochondral lesion, osteophyte formation in femur, tibia and patella, and in- 
crease or decrease of articular space.

MRI was performed on sagittal and coronal planes using a 0.5 Tesla scanner. Three imaging pulse sequences were used: a) T1weighted spin echo (SE) with a repetition time (RT) of $700 \mathrm{~ms}$, echo time (ET) of 25 $\mathrm{ms}(700 / 25)$, acquisition time of $2 \mathrm{~min}$ and $59 \mathrm{~s}$, and NEX of 2; b) T2-weighted SE with an RT of $2000 \mathrm{~ms}$, ET of $100 \mathrm{~ms}$ (2000/100), with an acquisition time of $8 \mathrm{~min}$ and $32 \mathrm{~s}$, and NEX of 2; c) proton density-weighted SE with an RT of $2000 \mathrm{~ms}$, ET of $25 \mathrm{~ms}$ (2000/25), with an acquisition time of $8 \mathrm{~min}$ and $32 \mathrm{~s}$, and NEX of 2 . The other parameters used were the same for all sequences: $15-\mathrm{cm}$ field of view and 3-mm thick sections with a 3-mm intersection gap and a $224 \mathrm{x}$ 128 matrix. An extremity coil was used. Each MR image was examined for the presence of femoral condyle osteochondral lesion, tibial osteochondral lesion, fibular osteochondral lesion, femoropatellar joint osteochondral lesion, osteophyte formation in femur, patella and femoropatellar joint, and increase or decrease of synovial fluid. One reader evaluated the XR images and another one evaluated the CT and MRI images. All exams were printed on radiological films. The readers did not know which limb was immobilized. The same scoring system was used to quantify degenerative joint disease for all the diagnostic methods. This system was based on a previous score reported by Vasseur and Berry (9). Each finding was assigned a value of 0 (absence), 1 (mild), 2 (moderate) or 3 (severe) depending on the severity of the changes and the values were summed to obtain a cumulative osteoarthritis score.

A macroscopic study was performed to obtain reliable reference data as a "gold standard" and to determine the accuracy of the diagnostic test XR, CT and MRI. The distal end of the femur and the proximal end of the tibia were separated, and the gross appearance of the joint surfaces and the periarticular soft tissues was evaluated. The ar- ticular cartilage was observed for loss of brightness, irregularities or presence of ulcers. Ligaments and the joint capsule were evaluated for rupture and thickness.

Data for immobilized and non-immobilized contralateral knees at TII, and between TI and TII for both knees were analyzed statistically by the nonparametric Wilcoxon rank sum test. Friedman repeated measures analysis of variance on ranks was used to compare the three methods of diagnosis (XR, CT and MRI) after 12 weeks of immobilization (TII) using the scores obtained for each one. Non-immobilized contralateral knees from the immobilized group and all knees from the control group were compared at TII by the nonparametric Mann-Whitney rank sum test. Differences were considered to be significant when the $\mathrm{P}$ value was $<0.05$.

\section{Results}

The osteoarthritis scores obtained by the three diagnostic methods are presented in Table 1.

Table 1. Total scores obtained with the three diagnostic methods based on a previous score reported by Vasseur and Berry (9).

\begin{tabular}{|c|c|c|c|c|c|c|c|c|c|c|c|c|}
\hline \multirow[t]{3}{*}{ Animals } & \multicolumn{4}{|c|}{ Radiography } & \multicolumn{4}{|c|}{ CT } & \multicolumn{4}{|c|}{$\mathrm{MRI}$} \\
\hline & \multicolumn{2}{|c|}{$\mathrm{TI}$} & \multicolumn{2}{|c|}{ TII } & \multicolumn{2}{|c|}{$\mathrm{TI}$} & \multicolumn{2}{|c|}{ TII } & \multicolumn{2}{|c|}{$\mathrm{Tl}$} & \multicolumn{2}{|c|}{ TII } \\
\hline & 1 & $\mathrm{NI}$ & I & $\mathrm{NI}$ & I & $\mathrm{NI}$ & I & $\mathrm{NI}$ & I & $\mathrm{NI}$ & I & $\mathrm{NI}$ \\
\hline 1 & 0 & 0 & 11 & 2 & 0 & 0 & 4 & 1 & 2 & 0 & 3 & 0 \\
\hline 2 & 0 & 0 & 5 & 1 & 0 & 0 & 4 & 1 & 1 & 0 & 4 & 0 \\
\hline 3 & 0 & 1 & 11 & 3 & 0 & 0 & 0 & 0 & 0 & 0 & 2 & 0 \\
\hline 4 & 1 & 0 & 9 & 3 & 0 & 0 & 16 & 6 & 0 & 0 & 11 & 1 \\
\hline 5 & 0 & 0 & 13 & 7 & 0 & 0 & 12 & 1 & 1 & 0 & 7 & 1 \\
\hline 6 & 1 & 1 & 11 & 2 & 0 & 0 & 10 & 2 & 0 & 0 & 6 & 1 \\
\hline 7 & 0 & 0 & 7 & 2 & 0 & 0 & 9 & 6 & 0 & 0 & 6 & 2 \\
\hline $\mathrm{Cl}$ & 1 & 2 & 4 & 2 & 0 & 0 & 0 & 0 & 0 & 0 & 0 & 0 \\
\hline $\mathrm{C} 2$ & 2 & 1 & 4 & 4 & 0 & 0 & 7 & 8 & 0 & 0 & 5 & 0 \\
\hline C3 & 0 & 0 & 4 & 2 & 0 & 0 & 1 & 1 & 0 & 0 & 0 & 0 \\
\hline
\end{tabular}

Each finding was assigned a value of 0 (absence), 1 (mild), 2 (moderate) or 3 (severe) depending on the severity of the changes and the values were summed to obtain a cumulative osteoarthritis score. I = immobilized limb; $\mathrm{NI}=$ non-immobilized contralateral $\operatorname{limb}$; $\mathrm{TI}=$ before immobilization; $\mathrm{TI}=$ after 12 weeks of immobilization; $\mathrm{C}=$ control group; $\mathrm{CT}=$ computed tomography; $\mathrm{MRI}=$ magnetic resonance imaging. 
Figure 1. Radiography of the right knee of a rabbit from the control group (A) and of an immobilized knee (B) showing patellar ligament mineralization (arrow). $A=$ rabbit $C 3$; $B=$ rabbit 1 (see Table 1).
Figure 2. Top: Sequence of $C T$ transverse planes (A, B) of knees from the control group (animal number 3). Femoral condyles (FC) are demonstrated. Bottom: Sequence of CT transverse planes (A, B) of immobilized and non-immobilized contralateral knees (animal number 2). The patellas (P) and FC can be observed. An osteophyte is evident on the medial condyle of the right (R) immobilized joint (arrows). $\mathrm{L}=$ left joint.

\section{Radiographic findings}

The data in Table 1 obtained before immobilization (TI) differed significantly from those obtained after 12 weeks of immobilization (TII) for both immobilized ( $\mathrm{P}=0.016$ ) and non-immobilized contralateral knees $(\mathrm{P}$ $=0.016)$. After 12 weeks, the immobilized

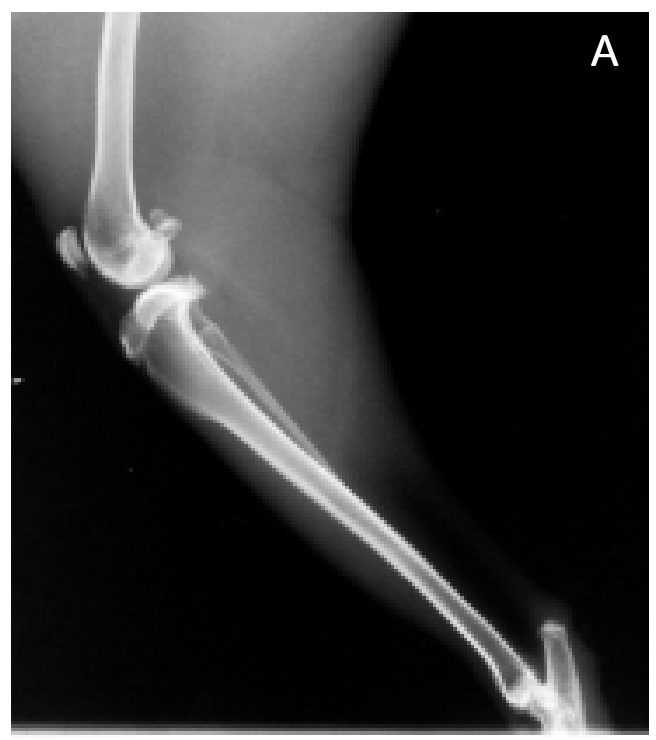

and non-immobilized contralateral knees showed different osteoarthritis scores $(\mathrm{P}=$ 0.016), whereas the scores for non-immobilized contralateral knees and control knees were not significantly different $(\mathrm{P}=0.295)$. A decrease of articular space was the most common change observed, also detected in the control group. The least common changes

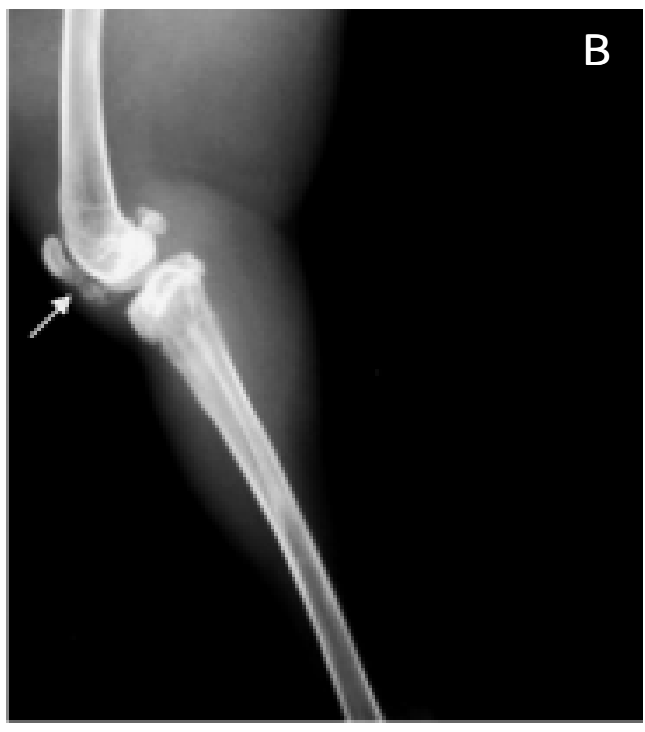

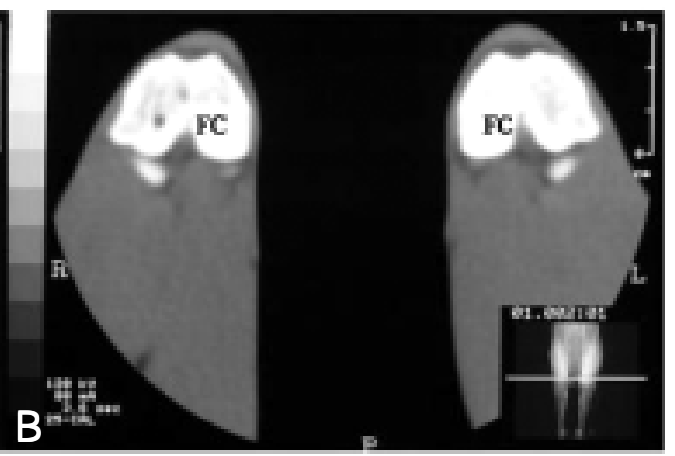
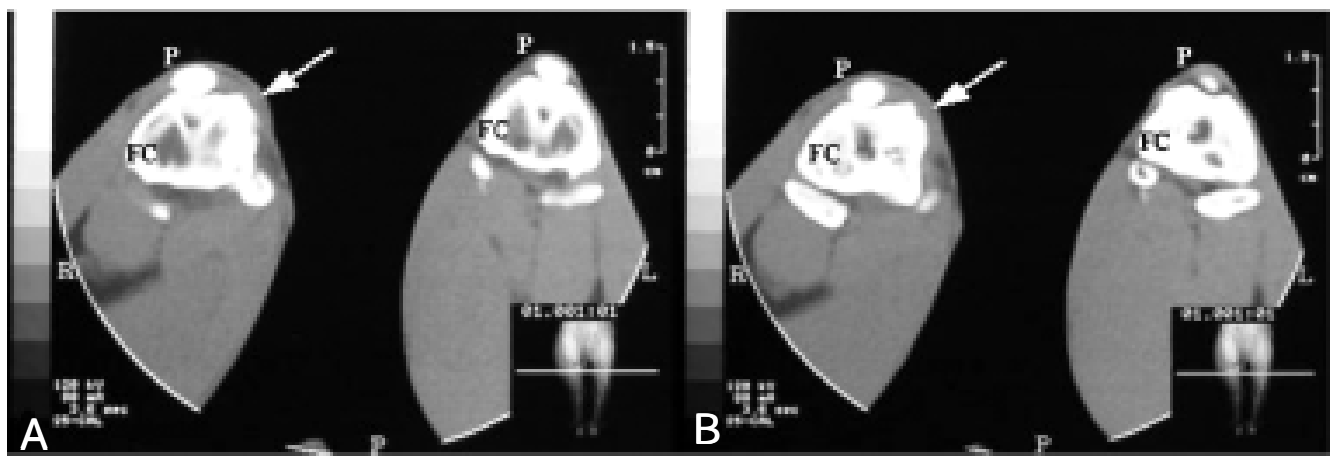
were femoral subchondral lesions and osteophyte formation in the tibia and trochlear groove. The control group did not show osteochondral reactions or osteophyte formation in the femoral condyles or patella (Figure 1A). Other changes were seen in some animals, such as femur head subluxation in non-immobilized contralateral limbs (four rabbits), muscle group asymmetry between immobilized and non-immobilized contralateral limbs (all immobilized rabbits) and patellar ligament mineralization (one rabbit; Figure 1B).

\section{Computed tomography findings}

Immobilized knees and non-immobilized contralateral knees were significantly different $(\mathrm{P}=0.031)$ after 12 weeks of immobilization (Table 1). There was a significant difference between TI and TII for both immobilized $(\mathrm{P}=0.031)$ and non-immobilized contralateral knees. However, there was no difference between the non-immobilized contralateral knees and the control group knees. No animal showed osteochondral lesions in the fibula and patella or osteophyte formation in the patella. Nevertheless, osteochondral lesions in femur, tibia and trochlear groove were the most common changes. The control group presented few alterations by $\mathrm{CT}$, like a mild decrease of articular space in the right and left limbs (Figure 2, top). Osteophytes were observed especially in the immobilized joint (Figure 2, bottom). Muscle asymmetry was visible upon CT examination in all immobilized animals.

\section{Magnetic resonance imaging findings}

Statistical analysis confirmed the difference between immobilized and non-immobilized contralateral limbs at TII $(\mathrm{P}=0.0156$, Table 1). The difference between TI and TII was significant for immobilized knees $(\mathrm{P}=$ 0.0156), but not for the non-immobilized contralateral knees. Comparison of the scores for the non-immobilized contralateral knees and control knees did not show significant differences. The animals showed some osteoarthritis alterations, the most common being osteochondral lesion (Figure 3A), but articular cartilage injuries could not be detected. The fibula and patella did not show any osteochondral change and increase of synovial fluid was observed in only two rabbits. One animal from the control group had a lesion revealed by MRI, but no changes were detected in the other two animals (Figure 3B). Some other alterations were soft tissue asymmetry between
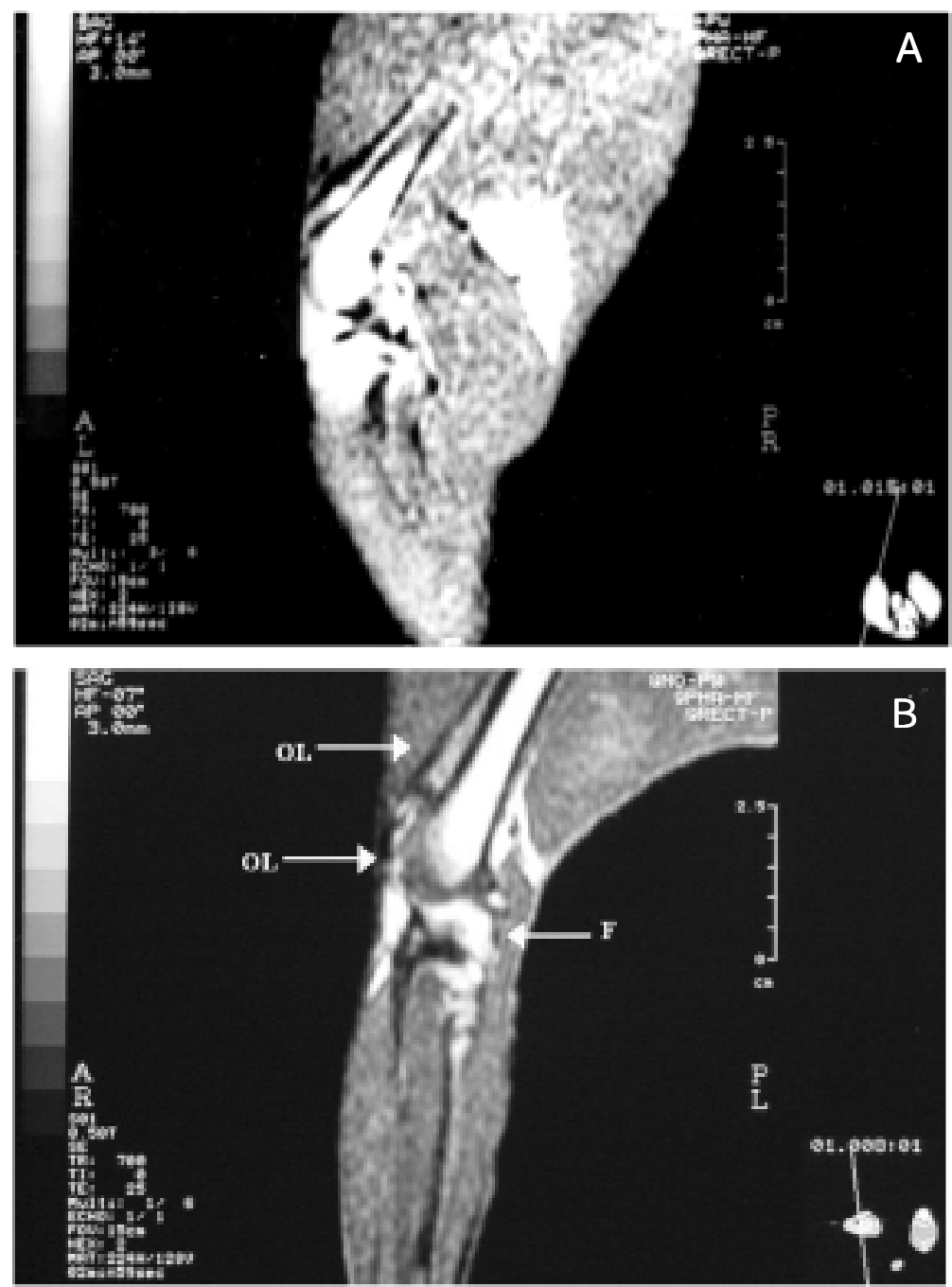

Figure 3. A, Sagittal plane of the right non-immobilized joint of an animal from the control group (animal number 3) in a T1-weighted spin echo sequence. B, Sagittal plane of an immobilized joint (animal number 5). Articular fluid (F) and osteochondral lesion (OL) in the femoral condyle and in the proximal trochlear groove in a T1weighted spin echo sequence are observed. 
limbs noted in all animals, and fluid collection in soft tissue observed in two animals and better visualized in T2-weighted SE images. Meniscus and cruciate ligaments were not well defined by MRI.

\section{Macroscopic findings}

Macroscopic evaluation of the immobilized group showed connective tissue on the femoropatellar joint border, muscle asymmetry and increased thickness of the joint capsule, especially on immobilized limbs. The control group did not present an increased thickening of the knee capsule. Proliferated tissue on the proximal femoropatel-

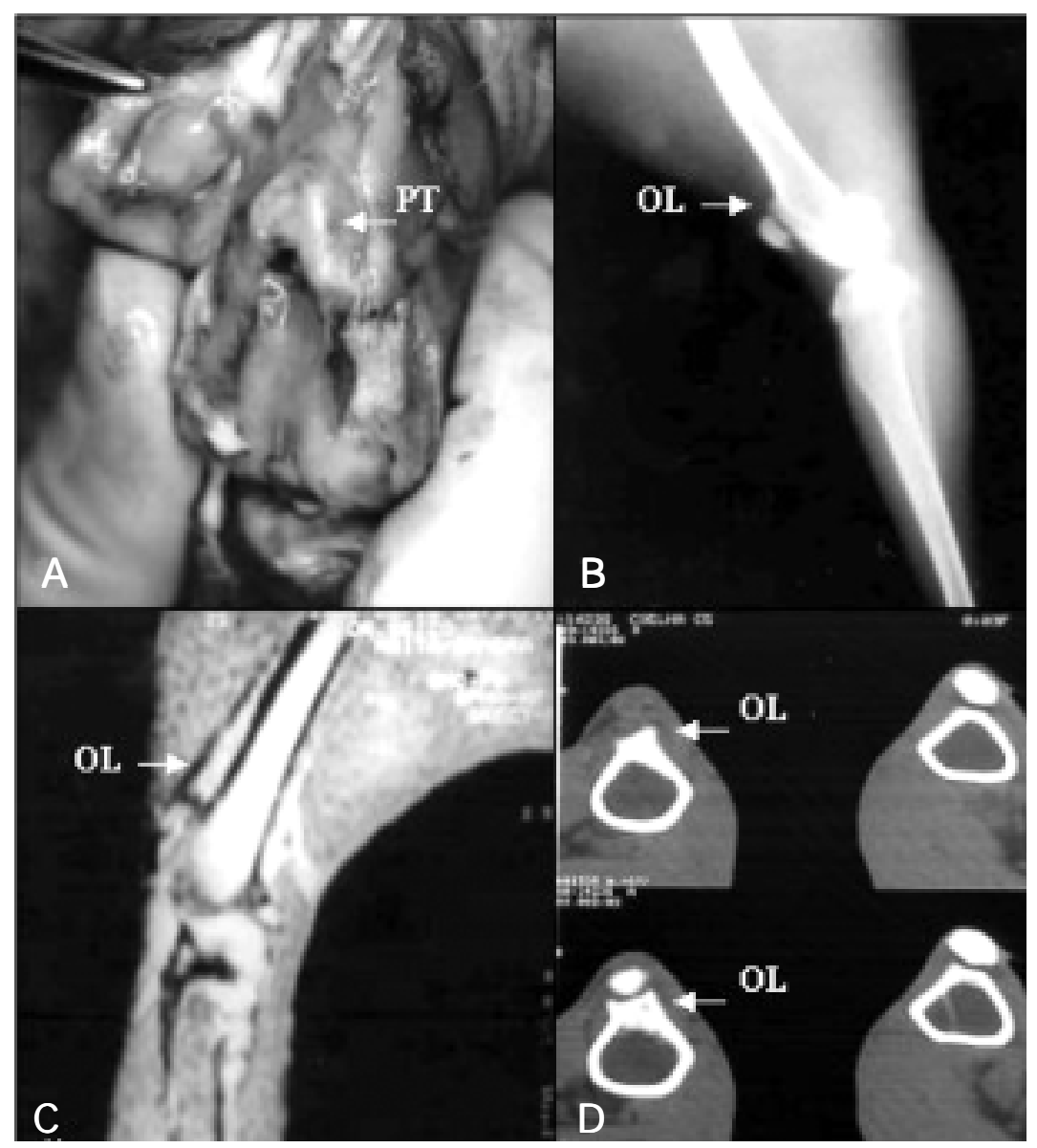

Figure 4. Proliferating tissue and osteochondral lesion in the proximal trochlear groove of an immobilized knee (animal number 5). A, Proliferating tissue (PT) observed by macroscopic examination; $\mathrm{B}$, osteochondral lesion (OL) observed by radiography; $\mathrm{C}$, OL detected by magnetic resonance imaging; $\mathrm{D}, \mathrm{OL}$ detected by computed tomography. lar joint and on the femoral condyle similar to cartilaginous tissue was more evident in immobilized knees (Figure 4). Ulcers on the articular cartilage were present in only one immobilized knee. Irregularities on the articular surface were very common in both immobilized and non-immobilized contralateral knees, especially fine irregularities associated with loss of brightness. Menisci did not show macroscopic changes and no ligament was ruptured or thick.

Muscle asymmetry was revealed by all imaging methods. The proliferated tissue on the proximal femoropatellar joint was demonstrated by the three methods (Figure 4), but only moderate to severe proliferation was visualized. Mild proliferated tissue was not detected by any method. Areas with proliferating tissue detected by macroscopic examination were associated with osteochondral lesion in imaging exams. Increased thickness of the joint capsule was not revealed by the imaging methods.

Comparison of the methods did not demonstrate a statistical difference among XR, $\mathrm{CT}$ and MRI in the immobilized knee; however, in the non-immobilized contralateral knees MRI 0.5 Tesla yielded a significantly higher score than XR and CT $(\mathrm{P}=0.027)$.

\section{Discussion}

The injuries observed in immobilized knees were compatible with osteoarthritis, as described in other studies (2). A wide variability of results was seen among animals for both joints, possibly due to differences in the extent of movement restriction produced by the splint (1).

Young animals have different abilities to repair joint lesions when compared to adults (11, apud 2). This is relevant information when we are comparing immobilized or nonimmobilized contralateral joints to observe osteoarthritis alterations. All animals had the growth plates almost or totally closed after the 12 weeks, so this fact probably did 
not have an effect on the observed lesions. Adult rabbits show abnormalities such as proliferating cartilage or osteophyte formation within 10 to 14 days of immobilization (3), findings that were detected here after 12 weeks or 84 days and that were not so marked. This was probably due to the fact that young rabbits were used.

Injuries present in non-immobilized contralateral knees were less severe than in the immobilized knees, but were clearly visible on the XR, which indicated small degenerative changes probably normal or due to mechanical stress from moving on three legs or to humoral factors (8). The method was appropriate to diagnose osteoarthritis in rabbits $(4,8,12)$. The XR permitted the observation of osteophytes in different parts of the knee, especially in immobilized joints (3). Osteophytes have been described as some of the most common alterations seen in dogs submitted to cranial cruciate ligament rupture, removal of medial meniscus or ligation of veins $(5,11,13)$. The joint space is important to characterize osteoarthritic joints $(3,4$, 11,13). This space includes synovial fluid with articular cartilage, joint effusion, ligaments, intracapsular fat, and menisci that make it difficult to assess joint space by a radiographic exam (4). However, the increase of articular space present in three animals could be associated with joint effusion.

$\mathrm{CT}$ is considered to be a good method for demonstrating morphological and structural changes of the knee anatomy without any kind of contrast (6). It was possible to differentiate the immobilized and non-immobilized contralateral knees by CT. The most common changes observed by $\mathrm{CT}$ in immobilized and non-immobilized contralateral knees were the osteochondral lesions, but less severe lesions were present in the nonimmobilized contralateral knees, just as observed on the XR. The proliferating tissue in proximal femoropatellar joint and femoral condyles was better visualized by CT than by XR because of the elimination of super- imposed structures (4). Cruciate ligament alterations were not evident on the axial plane. Sagittal and coronal reconstructions, which are described as the better views for this ligament (6), were not performed. Demonstration of meniscal tears was not possible, but advances in surface coils and a better resolution of imaging permit the diagnosis of hyaline cartilage degeneration, meniscal tears and meniscal degeneration (14).

MRI permits a direct visualization of all components of the joint simultaneously and can detect a lot of joint abnormalities (4), but in the present study, in contrast to other reports, it could not identify changes in articular cartilage. Probably the cartilage alterations were not severe enough to be evident by the MRI techniques used in the present study although they were demonstrable by macroscopic examination. Clinical and research studies have yielded contradictory and disappointing data concerning the detection of cartilage lesions by MRI (15). There are other techniques for evaluation of the joint and especially for the detection of cartilage changes. CHESS and FLASH sequences can be used to study articular cartilage because these techniques permit better differentiation between fluid and watery tissues, like hyaline cartilage and muscle with high signal intensity, and fatty tissues, like bone marrow and subcutaneous fat with a low signal intensity (14). Other techniques include the fat-suppressed sequences, that are significantly better to identify cartilage lesion, and other articular structures in arthritic knees, when compared to the spinecho sequences and the three-dimensional gradient-recalled echo sequences (gradientrecalled acquisition in the steady state GRASS and spoiled GRASS - SPGR) with no fat-suppression $(15,16)$. A 0.5 Tesla scanner was used for this study, which provides a lower spatial resolution compared to 1.5 Tesla scanners that have been used in most of the other studies. Sequences that permit better 
differentiation of cartilage, like the fat-suppressed ones, were not determined because of the limitation of the scanning technique. With MRI it was possible to differentiate immobilized and non-immobilized contralateral knees, as done by XR and CT. Osteochondral lesions were also the most common alterations observed by MRI. Joint effusion was observed on T2-weighted SE, a sequence that permitted a better differentiation of the surrounding musculature $(7,12)$.

Osteoarthritis changes were seen in both immobilized and non-immobilized contralateral knee joints, in agreement with earlier studies about articular degeneration resulting from immobilization $(2,8)$. However, the scores obtained for non-immobilized contralateral knees from the immobilized group did not differ from those for the control group, probably because the contralateral knees developed normal changes for rabbits of this age kept under these conditions. Degenerative injuries were observed in nonimmobilized joints evaluated by XR as described in other studies (8). Nevertheless, when transection of the right cranial cruciate ligament was used to induce osteoarthritis in dogs, radiographic injuries were not detected in the contralateral knee (5).

The lesions in the immobilized knee were compatible with osteoarthritis, leading to differentiation of the immobilized limb and confirming the efficiency of the induction method. The information obtained with each diagnostic method was complementary and helped to diagnose osteoarthritis, but no method detected all lesions present in the joints. The small number of readers must be considered to be a limitation of this study. XR was a good method for the assessment of bone changes, but did not have good contrast resolution and some structures were not well defined. CT had a better contrast resolution and could show bone lesions in more detail than XR. MRI had excellent contrast resolution, especially for soft tissue; however, it did not detect more changes than XR or CT. Immobilized knees did not show differences between the three diagnostic methods, but for the non-immobilized contralateral knees only XR and CT were compatible, probably because of mild alterations observed in these joints, especially in soft tissue.

We conclude that the techniques used in the present study, XR, CT and MRI 0.5 Tesla scanner, similarly detected osteoarthritis lesions in rabbit knees. XR and CT showed more bone osteoarthritis changes than MRI with a 0.5 Tesla scanner. None of the methods revealed all the lesions detected by macroscopic examination, especially mild changes.

\section{References}

1. Sood SC (1971). A study of the effects of experimental immobilisation on rabbit articular cartilage. J ournal of Anatomy, 108: 497-507.

2. Paukkonen K, J urvelin J \& Helminen HJ (1986). Effects of immobilization on the articular cartilage in young rabbits - a quantitative light microscopic stereological study. Clinical Orthopaedics and Related Research, 206: 270-280.

3. Langenskiöld A, Michelsson JE \& Videman T (1979). Osteoarthritis of the knee in the rabbit produced by immobilization. Acta Orthopaedica Scandinavica, 50: 1-14.

4. Carrig CB (1997). Diagnostic imaging of osteoarthritis. Veterinary Clinics of North America. Small Animal Practice, 27: 777-813.

5. Widmer WR, Buckwalter KA, Braustein EM, Hill MA, O'Connor BL \& Visco DM (1994). Radiographic and magnetic resonance imaging of the stifle joint in experimental osteoarthritis of dogs. Veterinary
Radiology and Ultrasound, 35: 371-383.

6. Passariello R, Trecco F, De Paulis F \& De Amicis R (1983). Computed tomography of the knee joint: clinical results. J ournal of Computer Assisted Tomography, 7: 1043-1049.

7. Hartzman S, Reicher MA, Bassett LW, Duckwiler GR, Mandelbaum B \& Gold RH (1987). MR imaging of the knee. Part II. Chronic disorders. Radiology, 162: 553-557.

8. Videman T (1982). Experimental osteoarthritis in rabbit. Acta Orthopaedica Scandinavica, 53: 339-347.

9. Vasseur PB \& Berry CR (1992). Progression of stifle joint osteoarthrosis following reconstruction of the cranial cruciate ligament in 21 dogs. J ournal of the American Animal Hospital Association, 28: 129-136.

10. Volpi RS (1989). Padronização de modelo experimental de artropatia 
induzida pela imobilização contínua do joelho em coelhos. Master's thesis, Departamento de Cirurgia e Anestesiologia Veterinária, Faculdade de Medicina de Botucatu, Universidade Estadual Paulista, Botucatu, SP, Brazil.

11. Shapiro F \& Glimcher MJ (1980). Induction of osteoarthrosis in the rabbit knee joint. Clinical Orthopaedics and Related Research, 147: 287-295.

12. Videman T, Eronen I \& Friman C (1981). Glycosaminoglycan metabolism in experimental osteoarthritis caused by immobilization. Acta Orthopaedica Scandinavica, 52: 11-21.

13. Tirgari M (1978). A study of the effects of various experimental surgical procedures designed to produce degenerative arthritis in dogs. J ournal of the American Animal Hospital Association, 14: 757-765.
14. Konig H, Sauter R, Deimling M \& Vogt M (1987). Cartilage disorders: comparison of spin-echo, CHESS, and FLASH sequence MR images. Radiology, 164: 753-758.

15. Recht MP, Kramer J M Marcelis S, Pathria MN, Trudell D, Haghighi P, Sartoris DJ \& Resnick D (1993). Abnormalities of articular cartilage in the knee: analysis of available MR techniques. Radiology, 187: 473-478.

16. Peterfy CG, Majumdar S, Lang P, Van Dijke CF, Sack K \& Genant HK (1994). MR imaging of the articular knee: improved discrimination of cartilage, synovium, and effusion with pulsed saturation transfer and fat-suppressed T1-weighted sequences. Radiology, 191: 413419. 